\title{
Ampullary adenocarcinoma: the current state of adjuvant therapies
}

\author{
Charles C. Vining, Darryl Schuitevoerder, Kiran K. Turaga \\ Section of General Surgery/Surgical Oncology, The University of Chicago Medicine, Chicago, USA \\ Correspondence to: Kiran K. Turaga. Section of General Surgery/Surgical Oncology, The University of Chicago Medicine, Chicago, USA. \\ Email: kturaga@surgery.bsd.uchicago.edu. \\ Comment on: Ecker BL, Vollmer CM Jr, Behrman SW, et al. Role of Adjuvant Multimodality Therapy After Curative-Intent Resection of Ampullary \\ Carcinoma. JAMA Surg 2019;154:706-14.
}

Submitted Nov 20, 2019. Accepted for publication Dec 14, 2019.

doi: 10.21037/hbsn.2019.11.37

View this article at: http://dx.doi.org/10.21037/hbsn.2019.11.37

Periampullary cancers, including adenocarcinoma from the duodenum, pancreas, bile duct and ampulla, represent a spectrum of malignancies with varying biological behavior and outcomes. In particular ampullary adenocarcinoma (AAC) is a rare, poorly understood disease with an incidence of $<1 / 100,000$ cases, compromises only $0.2 \%$ of gastrointestinal cancers and is frequently grouped together with other periampullary cancers (1). Five-year survival of resected AAC varies from $30-70 \%$ and is primarily thought to be due to the histologic subtype based on the epithelium of origin (intestinal, pancreaticobiliary or mixed-type) and subsequent tumor biology $(2,3)$. Additionally, AAC has been demonstrated to have somatic and germline genetic alterations and molecular subclassifications which makes it evident that AAC represents a spectrum of disease. Wholeexosome sequencing and DNA copy-number analysis of patients with AAC has identified 24 driver genes that are recurrently mutated including TP53, KRAS, CTNNB1 and SMAD4 seen in both subtypes (4). Intestinal AAC mutations are more similar to those in colorectal cancer with increased alterations in the $W N T$ pathway whereas the pancreaticobiliary subtype is more akin to pancreatic cancer with increased $K R A S$ and $T P 53-R b$ signalling. Additionally, actionable mutations such as BRCA2, ATM, KRAS, MDM2, $P I 3 C K A$, and $E R B B 2$ have been discovered in AAC and may prove to have therapeutic benefits (5).

Currently, localized disease standard of care is complete surgical excision with pancreaticoduodenectomy (PD) with limited and conflicting data to support or refute adjuvant therapy (AT). Fortunately, AAC is more frequently resectable at the time of diagnosis due to its location and propensity to cause early biliary obstruction or pancreatitis and thus earlier detection with resection rates reaching $50 \%(3,6)$. This is in stark contrast to the vast majority of pancreas adenocarcinomas which are unresectable at the time of diagnosis. This phenomenon in addition to the more favorable biology of some AAC portends a better prognosis when compared to pancreatic cancer and cholangiocarcinoma. Some have advocated for transduodenal ampullectomy for early stage disease but given the risk of nodal metastasis exceeding $20 \%$ for T1 disease, PD remains the favored approach (7). Moreover, given the scarcity of the disease, data pooling of periampullary cancers and counterpoise of data, there are no published formal treatment consensus guidelines. Therefore, the decision to pursue adjuvant treatment is based on individualized expert clinical opinion.

There have been at least 4 randomized clinical trials and several other retrospective studies that have evaluated the effect of AT on periampullary cancer including AAC. The ESPAC-3 randomized controlled trial evaluated the effect of AT in patients with periampullary cancer following PD. Although there was no significant difference in the primary analysis, after adjustment, adjuvant chemotherapy compared with observation conferred a statistically significant survival benefit (HR, 0.75; 95\% CI, 0.57-0.98; $\mathrm{P}=0.03$ ) (8). The EORTC 40891 trial evaluated patients with pancreatic and periampullary cancer and randomized them to postoperative chemoradiotherapy versus observation following resection and found no difference in overall survival (OS) (9). Takada et al. reported on postoperative adjuvant chemotherapy with mitomycin $\mathrm{C}$ and 5-FU versus surgery 
alone in patients with resected pancreatic, bile duct, gallbladder or ampulla of vater carcinomas. There was no significant difference in 5-year survival or 5-year disease free survival (DFS) for patients with pancreatic, bile duct or ampulla of vater carcinomas, but the 5 -year survival (26.0\% vs. $14.4 \% ; \mathrm{P}=0.0367)$ and 5 -year DFS $(20.3 \%$ vs. $11.6 \% ; \mathrm{P}=0.0210)$ in patients with gallbladder cancer who underwent AT was statistically improved (10). Morak et al. reported their results of adjuvant celiac axis infusion and radiotherapy in patients with resected pancreatic or periampullary cancer. Patients were randomized to adjuvant intra-arterial mitoxantrone, 5-FU, leucovorin and cisplatinum with $30 \times 1.8$ Gy radiotherapy. In patients with periampullary carcinomas there was no significant difference in OS or locoregional recurrence with AT, but this group of patients developed less liver metastasis (11). A meta-analysis by Kwon et al. included 3,361 patients with periampullary cancer and demonstrated a survival benefit of adjuvant chemoradiation following PD, especially for patients with T3/T4 tumors and those with positive lymph nodes (12). Moekotte et al. evaluated independent predictors of OS in 887 patients who underwent PD for AAC from 9 European tertiary referral centers. Nodal stage, perineural invasion (PNI) and adjuvant chemotherapy were independent predictors of OS in multivariable analysis (13). Several other small retrospective studies have found survival advantage of adjuvant chemoradiotherapy for AAC following PD. Therefore, the data is inconclusive with regards to the benefit of $A T$ in resected AAC.

Ecker et al. elegantly report their findings in a recent issue of JAMA Surgery where 357 patients with AAC from 12 institutions which make up the Ampullary Carcinoma Study Group were retrospectively queried. As previously identified, patients with intestinal subtype AAC had an improved OS compared with those with pancreaticobiliary subtype (77 vs. 54 months). Node positivity and PNI were associated with receipt of AT, whereas older age ( $>75$ years old) and black race were associated with omission. Histologic subtype was not associated with receipt of AT. In both the unmatched and propensity score matched cohorts, the addition of AT in the form of adjuvant chemotherapy, adjuvant radiotherapy or both, the overall survival was not significantly different compared to patients who underwent surgery alone. When dichotomized by histologic subtype (pancreaticobiliary $v s$. intestinal) and chemotherapy regimen (gemcitabine based, fluorouracil based or combination), there was no association between AT and OS. The addition of radiotherapy was not associated with improvement in OS or recurrence free survival in either subtype. In the entire cohort, there was no clear subgroup of patients in which AT was beneficial (14). The inability to determine which patients completed their course of AT is a significant limitation in this study. Additionally, only a minority of patients underwent multiagent chemotherapy, which may be more beneficial than single agent. With the increased risk of CR-POPF, those at higher risk disease who may be more likely to benefit from AT may never have received it. Finally, the Ampullary Carcinoma Study Group institutions could be more likely to see cases of greater complexity and therefore may not represent the population. Moreover, the majority of patients were Caucasian $(87.1 \%)$ and had stage III $(65.5 \%)$ disease, which again, may not represent the population.

With conflicting data and minimal progress in overall survival over the past several years, where do we go from here? The decision to pursue AT remains debatable and it is difficult to ascertain if this study adds anything to the current body of literature. Li et al. created the first validated ampullary carcinoma nomogram after surgery. Independent risk factors associated with survival after multivariate analysis included age, grade, tumor size, lymph node ratio, extension range and histology. The use of the nomogram may provide clinicians with an immediate and accurate tool following resection by which to decide on AT (15). The rate of clinically relevant postoperative pancreatic fistula is greater amongst those with AAC compared to those with non-ampullary adenocarcinoma. This association, driven primarily by soft gland texture, and pathologic subtype may limit AT and highlights the need for improved surgical technique. Recruitment is completed for a phase II clinical trial of patients with metastatic small bowel or ampulla of Vater adenocarcinoma to determine the progression free survival, response rate and overall survival in patients who receive oxaliplatin, capecitabine and bevacizumab (16). Despite advances in cancer care and research, it is clear that continued efforts are required to study and identify targets for this rare tumor. Improved surgical technique, neoadjuvant therapies and novel therapeutics may be the key to improved survival for patients with AAC.

\section{Acknowledgments}

Funding: None.

\section{Footnote}

Provenance and Peer Review: This article was commissioned 
by the editorial office, Hepatobiliary Surgery and Nutrition. The article did not undergo external peer review.

Conflicts of Interest: The authors have no conflicts of interest to declare.

Ethical Statement: The authors are accountable for all aspects of the work in ensuring that questions related to the accuracy or integrity of any part of the work are appropriately investigated and resolved.

Open Access Statement: This is an Open Access article distributed in accordance with the Creative Commons Attribution-NonCommercial-NoDerivs 4.0 International License (CC BY-NC-ND 4.0), which permits the noncommercial replication and distribution of the article with the strict proviso that no changes or edits are made and the original work is properly cited (including links to both the formal publication through the relevant DOI and the license). See: https://creativecommons.org/licenses/by-nc-nd/4.0/.

\section{References}

1. Kimura W, Ohtsubo K. Incidence, sites of origin, and immunohistochemical and histochemical characteristics of atypical epithelium and minute carcinoma of the papilla of Vater. Cancer 1988;61:1394-402.

2. Albores-Saavedra J, Schwartz AM, Batich K, et al. Cancers of the ampulla of vater: demographics, morphology, and survival based on 5,625 cases from the SEER program. J Surg Oncol 2009;100:598-605.

3. Rostain F, Hamza S, Drouillard A, et al. Trends in incidence and management of cancer of the ampulla of Vater. World J Gastroenterol 2014;20:10144-50.

4. Yachida S, Wood LD, Suzuki M, et al. Genomic Sequencing Identifies ELF3 as a Driver of Ampullary Carcinoma. Cancer Cell. 2016;29:229-40.

5. Wong W, Lowery MA, Berger MF, et al. Ampullary cancer: Evaluation of somatic and germline genetic alterations and association with clinical outcomes. Cancer 2019;125:1441-8.

6. Gonzalez RS, Bagci P, Basturk O, et al. Intrapancreatic distal common bile duct carcinoma: Analysis, staging considerations, and comparison with pancreatic ductal and ampullary adenocarcinomas. Mod Pathol 2016;29:1358-69.

7. Winter JM, Cameron JL, Olino K, et al. Clinicopathologic analysis of ampullary neoplasms in 450 patients: implications for surgical strategy and long-term prognosis.
J Gastrointest Surg 2010;14:379-87.

8. Neoptolemos JP, Moore MJ, Cox TF, et al. Effect of adjuvant chemotherapy with fluorouracil plus folinic acid or gemcitabine vs observation on survival in patients with resected periampullary adenocarcinoma: the ESPAC-3 periampullary cancer randomized trial. JAMA 2012;308:147-56

9. Smeenk HG, van Eijck CH, Hop WC, et al. Longterm survival and metastatic pattern of pancreatic and periampullary cancer after adjuvant chemoradiation or observation: long-term results of EORTC trial 40891. Ann Surg 2007;246:734-40.

10. Takada T, Amano H, Yasuda H, et al. Is postoperative adjuvant chemotherapy useful for gallbladder carcinoma? A phase III multicenter prospective randomized controlled trial in patients with resected pancreaticobiliary carcinoma. Cancer 2002;95:1685-95.

11. Morak MJ, van der Gaast A, Incrocci L, et al. Adjuvant intra-arterial chemotherapy and radiotherapy versus surgery alone in resectable pancreatic and periampullary cancer: a prospective randomized controlled trial. Ann Surg 2008;248:1031-41.

12. Kwon J, Kim BH, Kim K, et al. Survival Benefit of Adjuvant Chemoradiotherapy in Patients With Ampulla of Vater Cancer: A Systematic Review and Meta-analysis. Ann Surg 2015;262:47-52.

13. Moekotte AL, Lof S, Van Roessel S, et al. Histopathologic Predictors of Survival and Recurrence in Resected Ampullary Adenocarcinoma: International Multicenter Cohort Study. Ann Surg 2019. [Epub ahead of print].

14. Ecker BL, Vollmer CM Jr, Behrman SW, et al. Role of Adjuvant Multimodality Therapy After CurativeIntent Resection of Ampullary Carcinoma. JAMA Surg 2019;154:706-14.

15. Li HB, Zhao FQ, Zhou J. Prognostic Nomogram for Disease-Specific Survival in Patients with Non-metastatic Ampullary Carcinoma After Surgery. Ann Surg Oncol 2019;26:1079-85.

16. Overman, M. Bevacizumab, Capecitabine, and Oxaliplatin in Treating Advanced Small Intestinal or Ampulla of Vater Adenocarcinoma. ClinicalTrials.gov Identifier: NCT01208103.

Cite this article as: Vining CC, Schuitevoerder D, Turaga KK. Ampullary adenocarcinoma: the current state of adjuvant therapies. HepatoBiliary Surg Nutr 2020;9(5):647-649. doi: 10.21037/hbsn.2019.11.37 